\title{
BUDDA: A New Two-Dimensional Bulge/Disk Decomposition Code for Detailed Structural Analysis of Galaxies ${ }^{1}$
}

\author{
R. E. de Souza, D. A. Gadotti, and S. dos Anjos \\ Departamento de Astronomia, Instituto de Astronomia, Geofísica e Ciências Atmosféricas, \\ Universidade de São Paulo \\ Rua do Matão, 1226 - Cid. Univers. CEP 05508-900, São Paulo - SP, Brasil; \\ ronaldo@astro.iag.usp.br,dimitri@astro.iag.usp.br, sandra@astro.iag.usp.br
}

\begin{abstract}
We present BUDDA (BUlge/Disk Decomposition Analysis), a new code devoted to perform a two-dimensional bulge/disk decomposition directly from the images of galaxies. The bulge component is fitted with a generalized Sérsic profile whereas disks have an exponential profile. No other components are included. Bars and other sub-structures, like lenses, rings, inner bars and inner disks, are studied with the residual images obtained through the subtraction of bulges and disks from the original images. This means that a detailed structural analysis of galaxies may be performed with a small number of parameters, and sub-structures may be directly studied with no a priori assumptions. As has been already shown by several studies, two-dimensional fitting is much more reliable than one-dimensional profile fitting. Moreover, our code has been thoroughly tested with artificial data and we demonstrate it to be an accurate tool for determining structural parameters of galaxies. We also show that our code is useful in various kinds of studies, including galaxies of, e.g., different morphological types, and inclinations, which also may be observed at different spatial resolutions. Thus, the code has a broader range of potential applications than most of the previous codes, which are developed to tackle specific problems. To illustrate its usefulness, we present the results obtained with a sample of 51 mostly early-type galaxies (but covering the whole Hubble sequence). These results show some of the applications in which the code may be used: the determination of parameters for Fundamental Plane and structural studies, quantitative morphological classification of galaxies and identification and study of hidden sub-structures. We have determined the structural parameters of the galaxies in our sample and found many examples of hidden inner disks in ellipticals, secondary bars, nuclear rings and dust lanes in lenticulars and spirals, and also wrong morphological classification cases. We now make BUDDA generally available to the astronomical community.
\end{abstract}


Subject headings: galaxies: fundamental parameters — galaxies: photometry — galaxies: statistics — galaxies: structure — techniques: image processing — techniques: photometric

\section{Introduction}

It is widely assumed that the light distribution in galaxies may be described by one or more components, which might be dynamically and/or chemically distinct. While most of ellipticals (E) may be photometrically described by only one major spheroidal component, lenticular (S0) and spiral (S) galaxies in general need at least two components: a spheroidal bulge and a flattened disk. However, in almost every object we can trace the presence of other relevant components, such as primary bars, secondary bars (in spirals and S0s), inner disks, lenses and rings, none of which have been as carefully studied as the bulges and disks. Although there is an unclear and poorly understood interplay between these components, which is not generally taken into account in most structural analyses, bulge/disk decomposition has been a major motif behind a number of important contributions to our understanding on galaxy formation and evolution. Examples include the Tully-Fisher relation (Tully \& Fisher 1977), the Fundamental Plane (Faber et al. 1987; Dressler et al. 1987; Djorgovski \& Davis 1987; Bender et al. 1992, hereafter FP), the correlation between the scale lengths of bulges and disks (Courteau et al. 1996; de Jong 1996b; dos Anjos \& Gadotti 2004) and the evolution of galaxy morphologies (Marleau \& Simard 1998). See also Peng et al. (2002) and Graham (2002a,b) for further and more recent examples.

Two important issues are related to the subject of bulge/disk decomposition: the fitting method and the mathematical functions used to describe each component. Generally speaking, there are two fitting methods, the one-dimensional (1D) and the two-dimensional (2D). In the 1D method, an azimuthally averaged surface brightness profile of the galaxy under study, or the major or minor axis profiles, is fitted by one or more components. This method has the advantage of being simple and fast, and works in low $\mathrm{S} / \mathrm{N}$ conditions. However, it has two major problems. Firstly, the procedure to obtain the galaxy profile may vary, and each procedure has its own drawbacks. In isophote fitting (Jedrzejewski 1987), which is commonly used, changes in ellipticity and position angle at consecutive isophotes turn the profile poorly defined, since it is extracted not along a straight line but an arc. This is especially true when a bar or a strong spiral arm is present. Alternatively, one may extract the profile

\footnotetext{
${ }^{1}$ Based on observations made at the Pico dos Dias Observatory (PDO/LNA - CNPq), Brazil
} 
along the galaxy major axis, which may be itself poorly defined, but in this case much of the information in the image is thrown away (Baggett et al. 1998). Moreover, minor axis profiles are also essential in this case since the bulge dominates the profile along this axis. Secondly, 1D fitting averages out in a complicate way non-axisymmetric components, like bars. It is possible, though, to obtain reasonable results with 1D fitting, as shown by Kent \& Glaudell (1989), which study the SB0 galaxy NGC 936 by means of 1D decomposition but also incorporating further intricate steps (see also Kent 1986). In 2D fitting, information in the whole image is used to build a model for each component and thus differences in the projected ellipticities and position angles become an advantage, since they help to constrain the parameters of the different components. Moreover, non-axisymmetric components are now taken into account. There are several examples in the literature of studies showing that the 2D method is much more reliable than the 1D (de Jong 1996a), retrieving more accurate structural parameters.

While there are several examples in the literature of studies which use 1D fitting (Baggett et al. 1998), only in the last decade 2D fitting algorithms become more popular, mainly because of the higher computational speed of today's machines. As examples, we may point out the algorithms developed by de Jong (1996a), by Simard (1998), and more recently by Peng et al. (2002) and Trujillo et al. (2001). Most of the available algorithms were developed to tackle specific problems, like, e.g., face-on late-type spirals (de Jong 1996a), distant galaxies (Simard 1998), or nearby galaxies observed with very high spatial resolution (Peng et al. 2002). On the other hand, it is a common practice to include several components other than the spheroid and the disk in an attempt to get better fits. In this way, one can model bars and, in the case of Peng et al. (2002), also nuclear point sources, inner disks and inner bars, and lenses. In the Peng et al. algorithm 40 free parameters are used, and in several cases a single component is fitted by various different functions.

A caveat behind this philosophy of performing structural analysis is related to the fact that we do not know how well a single general function could fit these sub-structures with a given accuracy. Therefore these fits might be subject to an unknown systematic effect. For instance, as some bars show an exponential profile, especially in late-type galaxies, and others have a flat light distribution, especially in early-type galaxies (Elmegreen 1996), with a steep outer drop, it is difficult to foresee how a single general function could be used to fit bars and give meaningful structural parameters. This is also the case for lenses and other sub-structures. In our code, we explore the alternative route of fitting the two major components, representing the disk and the bulge contributions, and to perform a detailed study of sub-structures in the residual images obtained after the subtraction of these major components. In principle, this procedure could allow us to study the minor structures and assess the possibility to describe them as families of functions. It should be noted, however, 
that residual images can also be obtained with 1D fits if one can derive reasonable estimates of the apparent axis ratios of the bulge and disk components. For instance, Buta et al. (1992) show a residual image of NGC 4622 based on 1D decomposition expanded to 2D using assumptions about its components projected shapes. Also, 2D bulge/disk decomposition is not the only way to bring out hidden features. Using Fourier decomposition (see, e.g., Considère \& Athanassoula 1988), Buta et al. (1992) were able to bring out remarkably well the amazing counter-winding spiral arms in NGC 4622. To a lesser extent, a similar analysis can be performed via unsharp masking and color maps (see, e.g., Erwin \& Sparke 2002, for a recent application).

Therefore, following these guidelines, we have developed a new and general purpose algorithm, BUDDA ${ }^{2}$ - which stands for BUlge/Disk Decomposition Analysis - devoted to perform a detailed 2D structural analysis in galaxy images. The code allows us to perform a direct study on sub-structures in galaxies by means of accurate residual images, besides determining structural parameters of the main components, namely the bulge and the disk. In Sect. 2 we present the details of how the algorithm works. In Sect. 3 we present a thorough analysis of the reliability of the algorithm using a set of artificial data images of galaxies having known structural parameters. In Sect. 4 we apply our algorithm to a sample of 51 galaxies and show the results. Finally, in Sect. 5 we finish with a summary and a brief discussion of our results and the potential applications of our code.

\section{The Algorithm}

From the point of view of our algorithm, a galaxy is simply an image composed by just two major components: the bulge and the disk. In order to extract the properties of these two components we need to describe them in a generic way and predict their expected contribution at each pixel of the observed image. For the disk we have adopted the exponential profile (Patterson 1940; Freeman 1970):

$$
I_{d}(r)=I_{0 d} e^{-r / h}
$$

where $h$ is the disk scale length, $I_{0 d}$ represents the central brightness and $r$ is the semi major axis of the elliptical isophotes having ellipticity $e_{d}$. Both components, the bulge and the disk, share a common center located at $x_{0}, y_{0}$, but the ellipticity $\left(e_{d}, e_{b}\right)$ and position angle

\footnotetext{
${ }^{2}$ See http://www.astro.iag.usp.br/ dimitri/budda.html where BUDDA is available to the astronomical community.
} 
$\left(P A_{d}, P A_{b}\right)$ are not necessarily equal. In principle, we expect that the information on the shape parameters of the disk is mainly sampled in the outermost isophotes of the galaxy, while the bulge is more influential in the innermost central region. Therefore, by tracking the subtle pixel variations due to changes in the isophotal shape going from the center to the external region the program is able to model both the bulge and disk isophotal shapes.

Bulges are usually described in the literature by the $r^{1 / 4}$ profile or de Vaucouleurs' function. Several examples of elliptical galaxies are known to accurately obey the de Vaucouleurs' law over as much as 10 intervals of magnitude in surface brightness. On the other hand, there are also several objects, including bulges, where this relation does not provide an accurate description. For these reasons we rather prefer the use of the so called Sérsic profile, which is a more generic mathematical expression that includes the de Vaucouleurs profile as a special case. Moreover, there is strong evidence that the Sérsic profile is more efficient in the empirical description of isophotal properties of early type galaxies (Caon et al. 1993). Therefore, we adopt the solution of describing the brightness profile of the bulge by using the relation

$$
I_{b}(r)=I_{0 b} 10^{-b_{n}\left[\left(r / r_{e b}\right)^{1 / n}\right]},
$$

where $r_{e b}$ is the effective radius, $I_{0 b}$ is the central brightness and $n$ is an index controlling the shape of the brightness profile. For $n=4$ we recover the de Vaucouleurs profile, while for $n=1$ we have the exponential profile. Therefore the index $n$ is an important quantity describing the radial variations of the brightness profile. The numerical constant $b_{n}$ is defined in such a way that the the effective radius, $r_{e b}$, contains half of the total bulge luminosity and its value is approximately given by $b_{n} \simeq 0.868 n+0.142$. At $r=r_{e b}, I_{b}=I_{e b}$, the bulge effective surface brightness. The coordinate $r$ indicates the semi-major axis of the bulge elliptical isophotes centered on $x_{0}, y_{0}$, having an ellipticity $e_{b}$ and a position angle $P A_{b}$.

The central brightness profile is known to be heavily affected by the atmospheric seeing. Therefore we have included in the code a circular gaussian smearing of the brightness profile controlled by a parameter associated with the seeing radius $\left(a_{s}\right)$. This is similar to what was already done by de Vaucouleurs \& Capaccioli (1979) and Capaccioli \& de Vaucouleurs (1983). Basically it affects only the very central region where the convolution with the original profile is able to flatten the central brightness distribution. But its inclusion is quite important because otherwise we would get very discrepant models in the central region, changing the optimum minimum $\chi^{2}$ solution. We have also included in the code a supplementary correction of the sky level. In fact, all images were sky corrected before applying the code. But the fact is that even in a careful analysis the sky subtraction could be quite important mainly in the outer region where the disk and bulge brightness profiles come 
close to zero. An oversubtraction of the sky contribution could easily mimic a truncation of the external region of the disk, while an undersubtraction can be misinterpreted as an extended disk. Therefore, we have included this correction term $\left(\Delta_{\text {sky }}\right)$ in order to verify if the sky correction was done properly or not. Whenever this correction was higher than a few percent of the sky level we have reanalized the sky subtraction procedure to verify the reasons behind such discrepancies. In some cases, the sky subtraction might be difficult to determine due to the projection of nearby stars, companion galaxies, or simply due to the large size of the galaxy in the frame that precludes a good sampling of sky regions. These worse cases, where we could not improve the sky subtraction procedure, have been removed from our analysis. Note that we have done a careful sky subtraction in the galaxy images studied in this paper (see Sect. 4) and that these images of our sample of galaxies generally allow for a proper determination of the sky background. Thus, the cases where the $\Delta_{s k y}$ term suggests that the sky subtraction may have failed were indeed very rare in this study.

It is also possible to fit edge-on galaxies. In this case, the disk ellipticity is not anymore a useful parameter and it is discarded by the code. Instead, it uses a vertical light distribution for the disk which follows van der Kruit \& Searle (1981), i.e., $\propto \operatorname{sech}^{2}\left(\mathrm{z} / \mathrm{z}_{0}\right)$, where $z_{0}$ is the isothermal scaleheight. Moreover, the ellipses describing the bulge and the disk components do not need to be necessarily perfect: boxy and disky isophotes are allowed, and the code looks for the best description. These are described by generalized ellipses and the ellipse index parameter $\gamma$ controls their shape: for $\gamma=1$ the ellipses are perfect, while for $\gamma>1$ the ellipses are boxy; disky ellipses have $\gamma<1$.

The user also has the option of considering an inner truncation in the disk and an outer truncation in the bulge. Note, however, that in this study we have not restricted the contributions of both bulge and disk in any region of the galaxies whatsoever. We have added these truncations as user specifications in the code to allow for the freedom of studying, for instance, disks with type II profiles (Freeman 1970). Any user should be very careful, though, in using these parameters, by making a priori assumptions that a given area of the galaxy is dominated by the bulge or the disk, as was often done with the 1D fits. These truncations may also work as a way to exclude certain regions from the $2 \mathrm{D}$ fit. We stress again that this has not been done in this paper. For instance, this is not what we have done to deal with galaxies with bars or other conspicuous components. To model such cases, we rely on the code's ability to distinguish bars and spiral arms from the bulge and the disk by means of differences in the position angle, ellipticity and luminosity profile. Unless these three constraints are all equal for, say, the bulge and the bar, the code is able to separate these components, as is revealed by the analysis of barred galaxies (see Sect. 4), in which the combined luminosity profile of the bulge and disk models falls below the observed profile for the galaxy only in the bar region. 
Altogether the code needs to determine a total number of 11 main parameters: $x_{0}, y_{0}$, $I_{0 d}, h, e_{d}, P A_{d}, I_{0 b}, r_{e b}, e_{b}, P A_{b}$, and $n$. The others, $a_{s}, \Delta_{s k y}$, the two $\gamma$ indices (one for bulge and one for disk), and the two truncation radii (the outer one for bulge and the inner one for disk) may be set constant in general. An initial guess for these parameters, together with an initial allowed range of variation, are provided by the user in an input file read by the program. If the range of a given parameter is initialized as zero the code understands that it is a fixed parameter that should be kept constant during the iteration process. This is a useful way of dealing with parameters that are difficult to be determined even by using the whole image information. For instance, if we have an almost circular component the position angle becomes badly constrained and difficult to be determined. In these cases we have opted to have an initial run of the code, keeping these parameters fixed, and then a second run was made leaving them unconstrained. The program also expects to receive from the user information about the noise amplitude of the image being analyzed. This is a crucial piece of information since the code will decide the best solution by calculating the $\chi^{2}$ of the overall fit of the model. This information comes in two ways: the flat noise level and the Poisson noise level. The former is a number in counts that represents the noise in flat regions of the image, which is dominated by contributions from the noise in the sky level and from residuals due to imperfections in the flatfield normalization. The flat noise level can be estimated by the user or by the code itself; the minimum valid count for the code will be the square root of the flat noise level. The Poisson noise level is kept to 1 if the user wants to assume that the noise in the galaxy image is purely Poissonic. The code may also verify if this is a correct assumption and change this level accordingly. To avoid complicated regions in the image the user can flag those regions dominated by stars, defects or other companion objects that could spoil the solution. This may be done with the IRAF ${ }^{3}$ task IMEDIT. Note that while only bright stars may interfere in the code's determination of the bulge and disk parameters, the fainter ones still contribute to the calculation of the $\chi^{2}$. Thus it is very important that the galaxy image be as free as possible from this kind of perturbation. Moreover, the maximum radius of the galaxy can also the informed so that the code doesn't try to find information outside the region where the object is present. At the end of this initial step the code selects all valid pixels that will be used during the fitting procedure.

The fits are done in raw intensity units and all pixels have the same weight. To obtain the optimum solution we have adopted the multidimensional downhill simplex method (Press

\footnotetext{
${ }^{3}$ IRAF is distributed by the National Optical Astronomy Observatories, which are operated by the Association of Universities for Research in Astronomy, Inc., under cooperative agreement with the National Science Foundation.
} 
et al. 1989). The iteration scheme works simply by testing all the possible combinations of the parameters in each step and choosing the one having the smallest $\chi^{2}$. In each step, the multidimensional volume along the n-dimensional parameter space is preserved. If a minimum is found along any given direction in the parameter space, the program performs a parabolic interpolation to improve the estimate for the next step. When a global minimum is found, the program does a final parabolic interpolation and estimates the error along any given parameter using the maximum $\chi^{2}$ variation allowed by the confidence level demanded by the user. Thus, it must be noted that the errors at each parameter determined by the algorithm can sometimes be overestimated. This is not related to the fact that the decomposition is a 2D one, but only because the code uses the maximum $\chi^{2}$ variation allowed. In the end, the code generates a file containing the expected image for each component that could be added and compared with the original data.

The code is available in two executable task files: GMODEL and BMODEL. The former is the one which determines the best bulge and disk models for the galaxy under study; the latter builds synthetic images from the results of the former.

\section{Tests}

To verify the reliability of the BUDDA algorithm we have performed a long series of tests. Firstly, the code was compiled on different machines (PCs and workstations) and under different operational systems (Linux and Unix). As there are small differences between Linux and Unix Fortran compilers, we have made small changes in the code so as to have proper versions for Linux and Unix. The different versions were tested and it was checked that they give the same results in any machine when applied to the same galaxy, whether a real object from our sample or a synthetic generated model.

To certify that the code reliably retrieves the right structural parameters of bulges and disks in galaxies, we have applied it to 41 synthetic galaxies. These were built with the BMODEL executable within BUDDA so that we know the galaxies' parameters a priori and can compare them with the results when we apply the GMODEL BUDDA executable to the galaxies' images. The synthetic galaxies are made of a bulge and a disk, with no other structural component such as a bar, since the main goal of the tests was to verify the reliability in the values obtained for the structural parameters of bulges and disks. While it is true that the complexity of real galaxies, which contain bars, spiral arms etc., may cause difficulties in the retrieving of the correct parameters, we have argued above that the code is able to distinguish between the surface brightness contributions from different components through constraints such as the ellipticity, position angle and luminosity profile. 
Thus, bars and spirals are not considered by BUDDA and then appear more conspicuously in the residual images. Moreover, when our code is applied on such more complex galaxies, the determination of the errors in the estimates of the structural parameters is made accordingly, since it takes into account the value of the $\chi^{2}$ for the fit (see Sect. 2). The code was applied exactly in the same way as it is done for real galaxies. The first guesses for the structural parameters were randomly chosen so that they can initially be quite different from the original value used in the simulation. No matter how far from the right value the first guess for a parameter may be, the code gradually tends to converge to the right value, within the expected error. This shows how robust the results from the code are, even if one doesn't have a good estimate about the morphological properties of the galaxy under consideration before the algorithm is applied.

We have done two kinds of tests. In the first one, a good spatial resolution image was simulated, similar to most of the studies using ground-based telescopes without active or adaptive optics. For these tests we have adopted a formal resolution of a two arcsec seeing in a galaxy image with a diameter of around 2 arcmin. The results we present in the next section are based on observations generally somewhat better than this. The tests show that this resolution is already good enough to determine structural parameters with excellent reliability (see below). To check how BUDDA works in worse cases (e.g., imaging of dwarf galaxies or galaxies at intermediate redshifts), we applied the same tests with a resolution two times poorer than that.

It is worth noting that in these tests there was no user intervention, as should be the case with real galaxies, when some actions are recommended to be taken in advance in order to maximize the code efficiency in retrieving accurate data. For instance, in real galaxies GMODEL should be applied several times, choosing different sets of initial values, to search for the best $\chi^{2}$ result. That precaution avoids the adoption of a solution that could be located in a local minimum. In some cases, one may initially fix some geometric parameters by other means; for instance, we could guess the initial position angle or ellipticity with the ELLIPSE task from IRAF. Then, after a first solution is found, one may leave these parameters free to vary in a second run, using the initial values as those of the solution of the first run. This is the recommended procedure, for instance, when the ellipticity is too small (around 0.1) and the position angle is difficult to determine. However, none of these actions was done in the tests, so that their results are actually a lower limit for the code's accuracy. Poissonic noise was added in some of the synthetic images but we found it to be a minor source of error, as long as the $\mathrm{S} / \mathrm{N}$ ratio is reasonably high (i.e., around 50 in the central regions of the galaxy).

The results from the tests are shown in Fig(s). 1 and 2 for the good resolution case, and Fig(s). 3 and 4 for poor resolution. From Fig. 1 we can observe that the reliability 
of the code is excellent in recovering the surface brightness, scale length and position angle both of the bulges, indicated by the open squares, and disks, indicated by the open circles. While there are some minor trends, the agreement is always within the estimated fitting error delivered by the program. The ellipticities of bulges and disks are more difficult to estimate and are subject to larger errors. The reason is that for a fixed value of ellipticity, within the formal error, we can always find a set of the other photometric parameters that will result in a final error close to the best model. Therefore, the final error is relatively insensitive to an error in the intrinsic ellipticity of the two components. Moreover, this effect tend to be worse when the bulge/disk ratio is larger than 1 , since in that case a change in the disk ellipticity practically has no impact in the total error budget of the model. Therefore, great care should be exercised when interpreting the ellipticity results. The ellipticity of the most eccentric bulges may be underestimated, but again is within the errors. This ellipticity issue may be easily corrected with the actions discussed in the above paragraph, by using, for instance, IRAF tasks to have an initial value based on the observed image. The solid symbols in Fig(s). 1d and 3d show how the code works in the cases when the ellipticities of bulge and disk are treated as in real galaxies. The Pearson correlation coefficient $\mathrm{R}$ in these figures are relative only to the solid symbols. In Fig. 2 we present the results regarding the Sérsic index and the bulge/disk ratios. For large values of $n$ the adopted seeing can cause an important variation in the estimated value. However, for the vast majority of galaxies, including Es, this effect is not important since that index is bound to have a lower value close to the de Vaucouleurs $(n=4)$ model. The good agreement also found in Fig(s). 3 and 4 , where we have tested the impact of having low spatial sampling, is encouraging.

To verify the error produced in the residual images by the differences in the input and retrieved synthetic models, we have subtracted the latter from the former in Fig. 5. The left panels show a case of good spatial resolution while the right ones show a case of poor resolution. They show that the discrepancy is restricted to the central region, which has a size of the order of the seeing. The right panels represent an especially bad fit when the ellipticities were not treated as must be done in real galaxies. Such a problem may be easily identified by the bipolar pattern left. The entire original synthetic galaxy occupies the whole frame in the upper panels. The brightness and contrast levels displayed were chosen as to enhance the differences.

Another interesting point regarding these tests is that the code is able to identify disks which are up to 6 times fainter than the bulge (see Fig(s). 2 and 4), although this value depends somewhat on the geometric differences between disk and bulge (in fact, our results below show that the code can be even more sensitive). In any case, this result reinforces the BUDDA finding of Gadotti \& de Souza (2003) on the absence of disks in the SB0 galaxies NGC 4608 and 5701, since the bulge/disk ratio in normal S0s is around 2 (Binney \& Merrifield 
1998).

As noted above, the errors determined by the algorithm may be sometimes overestimated. This seems to be especially true for the brightness and for the position angle. The $1 \sigma$ error bars for the surface brightness are typically around 0.75 mag. However, from Fig(s). 1 and 3, one sees that fiducial errors may be considered to be around 0.25 mag. In these figures, there are no error bars associated with the position angles because most of the time the algorithm is not able to estimate them reliably, a fact that is related to the ellipticity issue discussed above. There are no error bars also related to the bulge/disk ratios (Fig(s). 2 and 4 ), but this is because they are calculated via the model images.

\section{A Structural Analysis Study of 51 Galaxies}

In order to test BUDDA in real galaxies we have observed 51 galaxies at the Pico dos Dias Observatory (PDO/LNA - CNPq, Brazil). The only criterion these galaxies obey is to be brighter than around 14 in B, and emphasis was put on Es and S0s. The CCD observations were done with a 24 inch telescope having a focal ratio $\mathrm{f} / 13.5$, and using a thin back-illuminated CCD SITe SI003AB, with $1024 \times 1024$ pixels. The plate scale is 0.57 arcsec/pixel, resulting in a field of view of approximately $10 \times 10$ arcmin. The CCD gain was set at $5 \mathrm{e}^{-} / \mathrm{ADU}$ and the read-out noise on $5.5 \mathrm{e}^{-}$. All objects were observed in the $\mathrm{R}$ passband of the Cousins system. For each object, we have done 3 exposures of 300 seconds. The multiple exposures aim to ease cosmic ray removal. The data were calibrated with a set of standard stars from (Graham 1982) and corrected for atmosphere and Galactic extinction. The later correction was done using the maps of Schlegel, Finkbeiner \& Davis (1998). The standard processing of the CCD data includes bias subtraction, flatfielding and cosmetics. The first step in the sky subtraction was done by editing the combined images, removing the galaxy and stars. After that step we determined the mean sky background and its standard deviation $(\sigma)$. Then, we removed all pixels whose values were discrepant by more than 3 $\sigma$ from the mean background. A sky model was obtained by fitting a linear surface to the image, and this model was subtracted from the combined image. We finally removed objects such as stars and HII regions. All these procedures were done using the IRAF package. The observations were done in 6 nights in October 1997. Typical values for the seeing and zero point error are, respectively, 0.8 to 1.2 arcsec and 0.02 to $0.05 \mathrm{mag}$.

Besides testing BUDDA, these data also allow us to perform a detailed structural analysis of galaxies. Since our primary concern is to verify sub-structures in early-type galaxies, our sample contains mostly Es and S0s, becoming also suitable to Fundamental Plane studies. However, our sample spans the whole Hubble sequence, with some examples of Ss, which are 
useful to test BUDDA in galaxies with spirals, bars, dust lanes and morphological peculiarities. Figure 6 shows the results for all galaxies. For each of them we have obtained with BUDDA the structural parameters and artificial images of the model galaxy, as well as of the model bulge and disk, separately. Using the IRAF ELLIPSE task, we built the surface brightness profiles of the galaxy, of the model galaxy, and of the bulge and the disk, as well as ellipticity, position angle and the b4 Fourier coefficient profiles for the galaxy and for the model galaxy. The behavior of the b4 coefficient indicates how much the isophotes deviate from a perfect ellipse. A positive value for b4 indicates isophotes with a disky component, whereas a negative value indicates boxy isophotes. If $\mathrm{b} 4=0$, then the isophote is a perfect ellipse. This whole procedure allows us to evaluate the quality of the fit and other structural parameters of the galaxy. Moreover, we obtained residual images, subtracting from the galaxy the model images, to verify the presence of sub-structures. In Fig. 6 we show, from left to right, and from top to bottom:

- Original Images, passed through a median filter and converted to surface brightness, with the isophotal map (with a 0.5 mag interval between each contour level)

- Total Residual Images, i.e., Original Image divided by Complete Model $^{4}$

- Disk Residual Images, i.e., Bulge Model subtracted from Original Image

- Bulge Residual Images, i.e., Disk Model subtracted from Original Image

- Surface Brightness profiles in the R-broadband, in magnitudes per squared arcsecond, as a function of the radius, in pixels, where

- points with error bars represent the Original Image

- the short-dashed line represents the Disk Model

- the long-dashed line represents the Bulge Model

- the full line represents the Complete Model

- points with full line represent the Total Residual profile (original image - complete model +28$)$, in magnitudes per squared arcsecond

- ellipticity, position angle, and b4 Fourier coefficient profiles, where

- points with error bars give values from Original Images

\footnotetext{
${ }^{4}$ Note that the total residual image is a ratio image, in which discrepancies are emphasized.
} 
- the full line represents the Complete Model

Through a careful analysis of Fig. 6, one can identify and study several features which would otherwise remain hidden in the direct galaxy image. Some points must be stressed concerning the analysis of residual images. In the computer, one may always change the brightness and contrast levels to carefully identify what is real and what is spurious. To make the figures, these levels are chosen to highlight features one wants to point out. Due to seeing effects the first few pixels in the nucleus are always brighter than the model and this excess extends to a few more pixels because there are too few data points for the model to be accurate. Thus, analysis in the very inner parts of the galaxy is always dubious. A level of subjectivity still remains in this kind of analysis, and because of that, the existence of any sub-structure may be confirmed also through the analysis of the radial profiles. For instance, an inner disk or bar may reveal itself in surface brightness radial profiles as a bump. Some other signatures are described in the following subsections.

Regarding our results, a first point that should be mentioned is that the broadest classification one may do with our sample is to distinguish between galaxies with only one spheroidal component, and galaxies that contain also an exponential disk. Behind this apparently banal statement, there are elliptical galaxies, which should be pure spheroidals, but contain inner disks, and lenticular galaxies, which should have a disk, but do not show any discernible disk, either because of wrong classification or secular evolution (in the case of 1 SB0, namely NGC 2217). Moreover, we find several other cases of wrong morphological classification and many galaxies with hidden sub-structures. We discuss these generic cases in the following subsections. Table 1 presents our results, including the structural parameters found for each galaxy.

We have made an extensive search in the literature for similar estimates for the galaxies in our sample, but it turned out that only to one galaxy a 2D decomposition code has been applied before in the R broadband. This is NGC 7177, studied also by de Jong (1996a). Unfortunately, however, our bulge parameters can not be compared since de Jong a priori assumed an exponential bulge for this galaxy, while our results show that its bulge is better described by a Sérsic profile with $n=3.52$. On the other hand, the disk parameters may be compared. The agreement shown is good indeed: while de Jong's results point to $I_{0 d}=18.73$ $\mathrm{R}$ mag $\operatorname{arcsec}^{-2}$ and $h=15.99$ arcsec, BUDDA estimates $I_{0 d}=18.58 \mathrm{R} \mathrm{mag} \operatorname{arcsec}^{-2}$ and $h=12.97$ arcsec. It is interesting that, at least in this case, using a different bulge luminosity profile did not alter significantly the estimate of the disk parameters. Moreover, the fact that de Jong also used a model for the bar of this galaxy indicates that BUDDA was able to distinguish the contribution to the total luminosity from the bulge, disk and bar, without assuming any luminosity distribution for the bar, which is very encouraging. 


\subsection{Inner Disks in Ellipticals}

There are 23 galaxies in our sample classified as Es in the RC3 (de Vaucouleurs et al. 1991). From these, only 9 seem to us to be pure spheroids, since the fit is excellent with only the spheroidal component and the residual images do not show any sub-structure (except in the nucleus, where seeing prevents any analysis). Prototypical examples are NGC 822, 6578, 6958, 7507 and 7619. Others that fall in this category are: IC 4842, 4943, NGC 720, and 1199. But even on some of these examples some doubt may be cast regarding the presence of any more complex morphology. Burstein et al. (2001) suggest that all galaxies have disks but $20 \%$ of them are not forming stars and a small fraction are too faint.

Eight galaxies classified as Es do show inner discs. These may be identified directly by the code, which in this case finds the exponential profile. This is the case for e.g. NGC 1052 and 7192. In some cases, however, the disk is too faint or too small to be recognized by BUDDA but it appears clearly in the residual images. One must bear in mind that these inner disks are not necessarily exponential disks, which is what our code tries to fit. Examples of this case are ESO 115-G008, PGC 64863 and NGC 821. Other signatures of the inner disk are disky $(\mathrm{b} 4>0)$ inner isophotes and a distinct behavior of the ellipticity and position

angle radial profiles from the inner to the outer regions of the galaxy. Disky isophotes are a signature of inner disks because it was found that Es with disky isophotes rotate faster (Kormendy \& Djorgovski 1989). However, the isophote being disky only means that there is an excess of light along the major and minor axes of the galaxy, which makes it look pointy. Bars seen close to or face-on also may produce disky isophotes. When $\mathrm{b} 4<0$, the isophotes are boxy. This means that the galaxy has a deficiency of light along its main axes, which causes a rectangular shape. Boxy isophotes are also found in some Es, which have negligible rotation (Kormendy \& Djorgovski 1989). Finally, boxy isophotes are also related to bars, but when seen edge-on (Binney \& Merrifield 1998). Other Es with inner disks are: NGC 1209, 1700 and 7196.

The remaining 6 galaxies are examples of bad morphological classification and will be discussed below. In all these galaxies we have found a large exponential disk which puts them in the lenticular class. In the misclassification section there are also galaxies classified as S0s, but that are actually Es, some of them with inner disks or complex morphology.

\subsection{Sub-structure in Lenticular Galaxies and another SB0 without Disk}

There are 20 galaxies in our sample classified as S0s in the RC3. Eleven are genuine lenticulars, in which BUDDA determined the disk parameters, except in one, namely NGC 
2217. This galaxy apparently has a very strong bar that could have destroyed the pre-existent disk within the bar length, and may be another case as those (NGC 4608 and NGC 5701) discovered by Gadotti \& de Souza (2003); see also Athanassoula (2003). Note, however, that, while NGC 4608 and NGC 5701 have indeed strong bars, as denote by their Fourier $m=2$ component (Gadotti \& de Souza 2003), it remains to be seen that the bar in NGC 2217 is really strong. As pointed out by Buta \& Block (2001), bar strength can be defined in terms of tangential forces relative to mean radial forces, and the latter are certainly significant in galaxies with large bulges, such as NGC 2217. Note, furthermore, that the existence of conspicuous rings outside the bar in these galaxies may be an indication of the existence of an outer disk, as the bar is only able to consume the disk within it.

Three of the eleven genuine lenticular galaxies are strongly barred and there are also three more strongly barred S0/a galaxies in our sample. In many of these systems one encounters interesting sub-structures which do not appear in their direct images. In some cases, structural peculiarities may be seen and recognized in a careful analysis of the direct images, but are certainly better defined in the residual ones. In NGC 1326 and 2217, the inner ring surrounding the bar, already identified by de Vaucouleurs \& de Vaucouleurs (1964), appears more clearly in the residual image (for a detailed analysis of NGC 1326, see Buta et al. 1998). Surprising dust lanes are visible in NGC 1316 and 1947, while the residual image of NGC 7280 displays a strong lens. An extremely interesting case is NGC 128. This edge-on galaxy, which is a prototype of peanut-shaped bulges was fitted by our code using its edge - on capabilities (see Sect. 2). The other objects which fall in this category are: IC 5250A, NGC 467, 2293, 6673, and 7377. The bulge/disk luminosity ratios for the lenticulars in our sample are around 2 with some noticeable deviation cases, in agreement with Binney \& Merrifield (1998).

In the remaining 9 galaxies we haven't found a large disk. These are clearly Es rather than S0s and will be discussed as misclassification cases below.

\subsection{Wrong Morphological Classification}

To distinguish between an elliptical and a lenticular galaxy is a hard task with visual inspection only, especially for faint and/or distant galaxies. Thus confusion between Es and S0s is common and may badly interfere in studies of e.g. scaling laws and the origin of the Hubble sequence. This is a point where codes like BUDDA may play an important role given their higher reliability. In our sample, 6 Es were found to have large disks which means they are actually S0s: IC 1459, NGC 1172, 1549, 2305, 7145, and 7778. In 3 of them (IC 1459, NGC 1549 and 7145) a lens is clearly visible in the residual images. 
On the other hand, 9 galaxies classified as S0s were found to have no large disk, which puts them in the $\mathrm{E}$ bin. As was also the case for some genuine ellipticals, some of these 9 systems show a morphology more complex than a pure spheroid. Prominent cases are the disturbed morphology of NGC 7252, the inner disk in NGC 2271, and the lens in NGC 1553, whose ratio image displays also an inner spiral in the center. The last case is especially intriguing since lenses are common in S0s. Considering the results from our code, either this is a rare case of a lens in an elliptical galaxy, or (more likely) BUDDA was not able to find the disk in this lenticular galaxy simply because its disk has not the common properties of exponential disks generally found in other disk galaxies. Its disk could possibly be inner truncated or have a flat inner profile (type II profile). The fact that its lens is also very bright and has an unusual appearance may have prevented our code to naturally find reliable estimates for the disk structural parameters. Indeed, BUDDA finds a disk in this galaxy, but that is a very faint disk with unusually high error estimates. It is worth noting that, as the position angle and the ellipticity of the lens are very similar to those of the bulge (and possibly the disk), it is very hard to disentangle the contribution of the lens to the total luminosity of this galaxy without a priori assumptions. NGC 1553 is certainly a case in which a more detailed analysis is in order. The other misclassified Es are: IC 1919, NGC 541, 2205, 6849, 7289, and PGC 67633.

It is interesting to point out that from the 43 Es and S0s galaxies in our sample (as considered in the RC3) 15 were shown to be in the wrong morphological bin, i.e., $\approx 35 \%$. Moreover, it seems (not surprisingly) that it is equally easy to misclassify an elliptical as an S0 and the other way around.

\subsection{Sub-structure in Spiral Galaxies}

Eight galaxies in our sample have a morphological class later than S0 according to the RC3. In many of them hidden sub-structures emerge from the residual images. We point out: the bar and the strong spiral arms in NGC 1079; the amazing case of NGC 1291 with the nuclear bar and inner ring ${ }^{5}$ that only show out clearly in the residual images [but note that de Vaucouleurs (1975) already recognized these features]; the bar and inner ring of NGC 7177; the bar and the unusual spiral arms of NGC 7371, and finally the weird spiral structure of NGC 7824. This last case is especially puzzling because, in spite of the spiral structure, a

\footnotetext{
${ }^{5}$ This ring may be actually a lens. Since a lens is like a ring of low surface brightness amplitude (see Buta \& Combes 1996), when a declining bulge and disk background is removed the lens then becomes very ringlike. In this case this may be particularly true given the broad appearance of this structure (but note that it is connected with the ansae at the ends of the bar).
} 
model containing only a bulge is able to fit the surface brightness profile very well. Again, this may be a case where the disk can not be modelled as it is traditionally done, i.e., with a exponential disk. The inner spirals seem to be detached from the outer ones. And the latter have an unusual broad appearance. Bars also reveal themselves in radial profiles of ellipticity (monotonically growing) and position angle (constant). The other spirals in our sample are: NGC 1637, 7070A, and 7171.

It is worth noting that in cases like NGC 1079 and NGC 1291, where our images do not sample the outermost parts of the galaxy, a better result for the disk model might be achieved with deeper and larger images (especially because, in these examples, the fits are affected by the outer ring feature). Also, in NGC 7171, the bulge looks overly important at large radii, which may be causing the bright halo around the ratio image. This is a case in which a more realistic model may be accomplished at the expenses of some a priori assumptions, like e.g., keeping the Sérsic index constant or assuming an outer truncation for the bulge.

\subsection{Nuclear Activity and Companions}

There are 7 galaxies in our sample which are classified as AGNs at some level (e.g., Liner, Seyfert) in the Nasa Extragalactic Database ${ }^{6}$. They are: IC 1459, NGC 1052, 1316, 1326, 1553, 7177, and 7280. There are also 10 galaxies which are classified as multiple systems in the Leda Extragalactic Database ${ }^{7}$ or show a clear companion in our images. This does not mean galaxies in groups or clusters and does not exclude systems that are only due to projection. It only means that these galaxies have a close companion in the plane of the sky. These are: ESO 115-G008, IC 5250A, NGC 128, 467, 1052, 2293, 7070A, 7196, 7252, and PGC 67633. The only galaxy which is a member of both sub-samples is NGC 1052. We have not found any clear tendency in these 2 groups of galaxies that makes them distinct from the whole sample. Only two wrong classification cases have companions, which means that these cases may not be caused by some unknown tidal process that could slightly change the morphology of a galaxy.

\footnotetext{
${ }^{6}$ See http://nedwww.ipac.caltech.edu/

${ }^{7}$ See http://leda.univ-lyon1.fr/
} 


\section{Concluding Remarks}

We have thoroughly presented BUDDA, a code devoted for bulge/disk decomposition in galaxy images. As was shown in details above, its development and performance tests show that it is a very suitable tool for the structural analysis of galaxies. The parameters for the bulge and disk models can be retrieved with great confidence as well as the residual images obtained from them. Its simplicity to use and its broad range of applications add to its qualities.

Applying BUDDA on our sample of 51 galaxies we could check how it works concerning its many potential applications. One obvious use of the code is to obtain bulge and disk structural parameters to study e.g. the Fundamental Plane and other scaling relations. Such a study regarding the galaxies in our sample will appear in a forthcoming paper. Such relations may also be strengthened if wrong morphological classification cases are identified, which is reliably done with our code, as we showed in Sect. 4.3. Galaxies classified as Es, but actually being S0s, which was the case for 6 of our galaxies, may add to the spread in scaling relations. Obviously, the opposite is also true. In the FP, for instance, S0s have generally lower mass-to-light ratios than Es (Bender et al. 1992).

Another use of BUDDA, in which we've found it to be very powerful, is to reveal hidden sub-structures, especially in the inner regions of galaxies. In Sect(s). 4.1 and 4.3 we revealed inner disks in many elliptical galaxies, which may be the result of a recent merger. Or are they related to galaxy formation processes? BUDDA has found also several examples of inner spirals, inner and nuclear rings, as well as inner and secondary bars in S0s and Ss. In some cases a hint of them shows up in the direct images or in the profiles, but in others there is no a priori sign of them at all. These sub-structures, which appear conspicuously in the residual images, are relevant for e.g. dynamical studies, since they are related to non-circular orbits and Lindblad resonances. Moreover, these sub-structures may possibly be relevant to the long standing problem of fueling active galactic nuclei (Combes 2001), and may be directly studied in the residual images.

We thank the Pico dos Dias Observatory staff for support during the observing runs. Financial support from FAPESP grants 99/07492-7 and 00/06695-0 is acknowledged. We would like to thank the anonymous referee for many enlightening comments and helpful suggestions. 


\section{REFERENCES}

Athanassoula, E. 2003, MNRAS, 341, 1179

Baggett, W. E., Baggett, S. M., \& Anderson, K. S. J. 1998, ApJ, 116, 1626

Bender, R., Burstein, D., \& Faber, S. 1992, ApJ, 399, 462

Binney, J., \& Merrifield, M. 1998, Galactic Astronomy, CUP

Burstein, D., Saglia, R. P., Colless, M., Davies, R. L., McMahan, R. K., \& Wegner, G. 2001, in Galaxy Disks and Disk Galaxies, ASP Conf. Series, 230, 153

Buta, R., Alpert, A. J., Cobb, M. L., Crocker, D. A., \& Purcell, G. B. 1998, AJ, 116, 1142

Buta, R., \& Block, D. L. 2001, ApJ, 550, 243

Buta, R., \& Combes, F. 1996, Fund. Cosmic Phys., 17, 95

Buta, R., Crocker, D. A., \& Byrd, G. G. 1992, AJ, 103, 1526

Caon, N., Capaccioli, M., \& D’Onofrio, M. 1993, MNRAS, 265, 1013

Capaccioli, M., \& de Vaucouleurs, G. 1983, ApJS, 52, 465

Combes, F. 2001, in Advanced Lectures on the Starburst-AGN Connection, Proceedings of a conference held in Tonantzintla, Puebla, Mexico, 26-30 June, 2000. Edited by Itziar Aretxaga, Daniel Kunth, and Raúl Mújica. Singapore: World Scientific, 2001., p.223

Considère, S., \& Athanassoula, E. 1988, A\&A, 76, 365

Courteau, S., de Jong, R., \& Broeils, A. 1996, ApJ, 457, L73

de Jong, R. S. 1996a, A\&A, 118, 557

de Jong, R. S. 1996b, A\&A, 313, 45

de Vaucouleurs, G. 1975, ApJS, 29, 193

de Vaucouleurs, G., \& Capaccioli, M. 1979, ApJS, 40, 699

de Vaucouleurs, G., \& de Vaucouleurs, A. 1964, in: Reference Catalog of Bright Galaxies, Austin: University of Texas 
de Vaucouleurs, G., de Vaucouleurs, A., Corwin, H. G., Buta, R. J., Paturel, G., \& Fouque P. 1991, in: Third Reference Catalog of Bright Galaxies, Springer-Verlag, New York (RC3)

Djorgovski, S, \& Davis, M. 1987, ApJ, 313, 59

dos Anjos, S., \& Gadotti, D. A. 2004, submitted

Dressler, A., et al. 1987, ApJ, 313, 42

Elmegreen, D. M. 1996, in Barred Galaxies, ASP Conf. Ser., 91 (San Francisco: ASP); edited by R. Buta, D. A. Crocker \& B. G. Elmegreen, p.23

Erwin, P., \& Sparke, L. S. 2002, AJ, 124, 65

Faber, S., et al. 1987, in Nearly Normal Galaxies, ed. S. M. Faber (New York: Springer), 175

Freeman, K. C. 1970, ApJ, 160, 811

Gadotti, D. A., \& de Souza, R. E. 2003, ApJ, 583, 75

Graham, A. W. 2002, MNRAS, 334, 859

Graham, A. W. 2002, MNRAS, 334, 721

Graham, J. A. 1982, PASP, 94, 244

Jedrzejewski, R. I. 1987, MNRAS, 226, 747

Kent, S. M. 1986, AJ, 91, 1301

Kent, S. M., \& Glaudell, G. 1989, AJ, 98, 1588

Kormendy, J., \& Djorgovski, S. 1989, ARA\&A, 27, 235

Marleau, F. R., \& Simard, L. 1998, ApJ, 507, 585

Patterson, F. S. 1940, Harvard Obs. Bull., 914, 9

Peng, C. Y., Ho, L. C., Impey, C. D., \& Rix, H-W. 2002, AJ, 124, 266

Press, W. H., Flannery, B. P., Teukolsky, S. A. \& Vetterling, W. T. 1989, in Numerical Recipes, CUP

Schlegel, D. J., Finkbeiner, D. P., \& Davis, M. 1998, ApJ, 500, 525 
Simard, L. 1998, in Astronomical Data Analysis Software and Systems VII, A.S.P. Conference Series, Vol. 145, 1998, R. Albrecht, R.N.Hook and H.A. Bushouse, eds., p.108

Trujillo, I., Aguerri, J. A. L., Gutierrez, C. M., \& Cepa, J. 2001, AJ, 122, 38

Tully, R. B., \& Fisher, J. R. 1977, A\&A, 54, 661

van der Kruit, P. C., \& Searle, L. 1981, A\&A, 95, 105 
Fig. 1. - Comparison between the parameters of the 41 synthetic galaxies as retrieved by BUDDA in the case of good spatial sampling: (a) central surface brightness of the disk and effective surface brightness of the bulge in arbitrary magnitudes; (b) characteristic radius of the disk and effective radius of the bulge in arbitrary units; (c) position angle, and (d) ellipticity. Although in (a) and (b) the units are arbitrarily chosen, all values displayed here are similar to those found for nearby observed galaxies. Circles are for the disc and squares are for the bulge parameters. The error bars were not considered for clarity reasons but a typical error bar is shown in each panel. Note that the typical error bar considered is as determined by BUDDA, which is not necessarily similar to the scatter in this figure. For instance, in (a) it is possible to see that our code typically overestimates the errors for the intensities. In (c) there are no error bars, for the code typically does not find a meaningful error estimate for the position angles, since variations in these parameters do not cause significant changes in $\chi^{2}$. The Pearson correlation coefficient $\mathrm{R}$ of each test is also indicated. For (d), $\mathrm{R}$ is relative only to the solid symbols. These refer to those tests in which the first guesses for the ellipticities were not random, but determined from the results of the ELLIPSE task in IRAF, as is done for real galaxies.

Fig. 2.- Same as Fig. 1 but for (a) Sérsic index, and (b) the bulge/disk ratios in luminosity (circles) and size (squares).

Fig. 3.- Same as Fig. 1 but for poor spatial sampling. Typical error bars are as in the good sampling case (see Fig. 1).

Fig. 4.- Same as Fig. 2 but for poor spatial sampling. Typical error bars are as in the good sampling case (see Fig. 2).

Fig. 5.- Examples of the discrepancy between a synthetic galaxy built with BMODEL and the one retrieved by GMODEL within BUDDA. The upper left panel shows a case of good spatial resolution while the upper right one shows a case of poor resolution. The last is an example of a specially bad fit. Even so, the differences are restricted to the central region, since the entire original galaxy occupies the whole frame. The brightness and contrast levels displayed were chosen as to enhance the differences. The lower panels show in details the central region of each of the former images, respectively.

Fig. 6.- Results of the BUDDA algorithm applied in R-band CCD images for all the 51 galaxies in our sample. At the top it is shown the name of the galaxy, its morphological type according to the RC3 and the size of the images in arcseconds. See the text for further explanations. The remaining figures are available in the electronic edition of the Journal. The printed edition contains only a sample. 

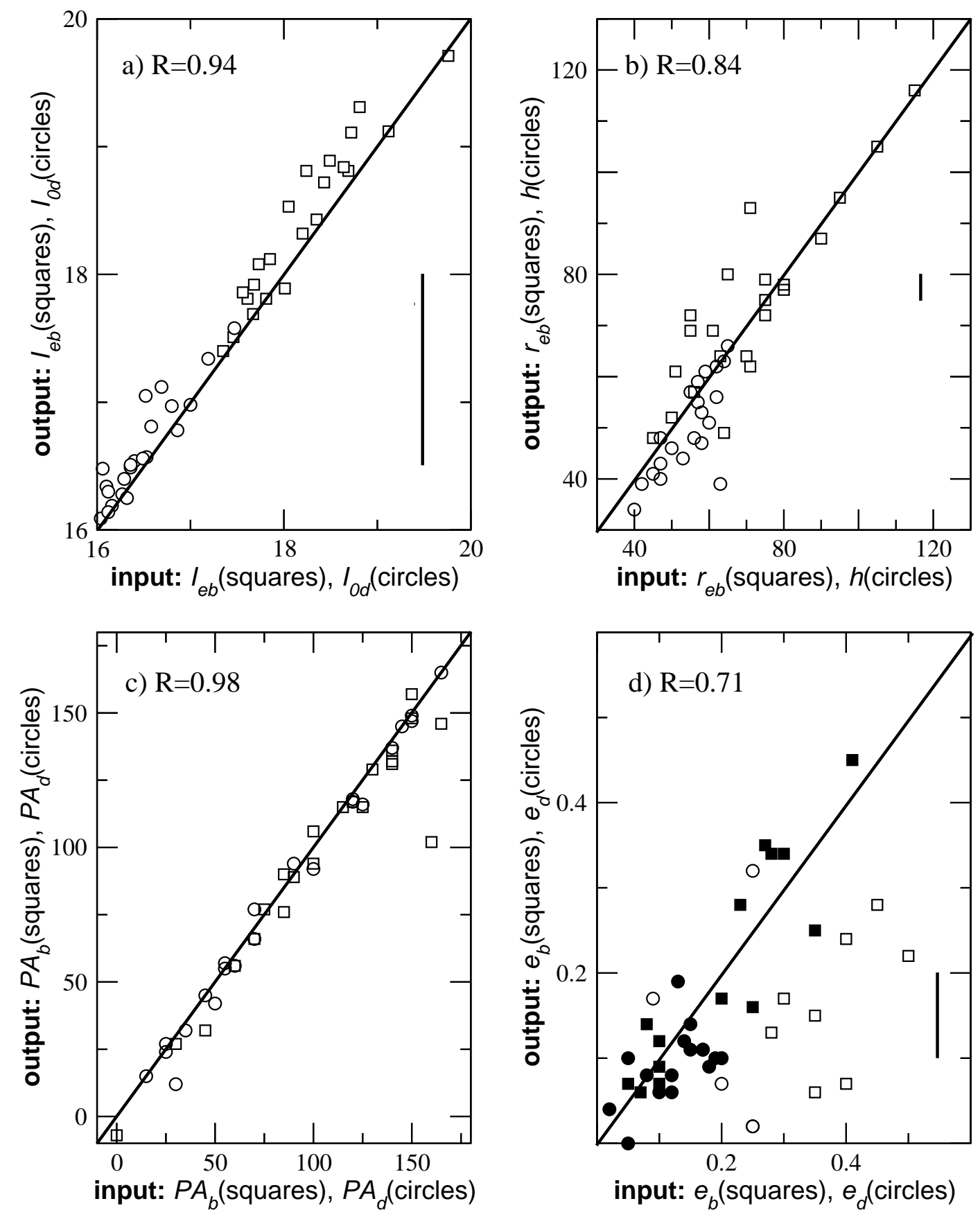

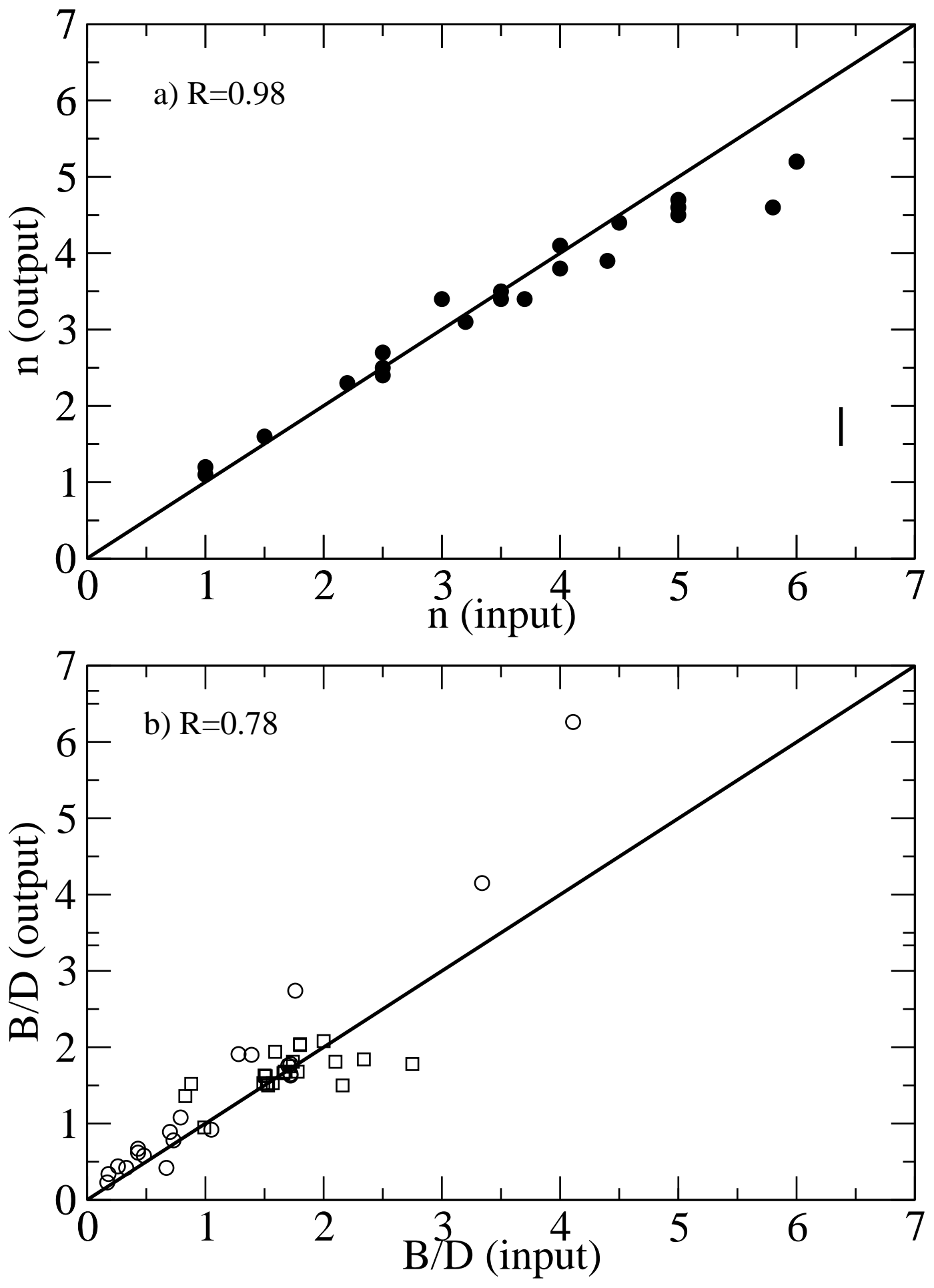

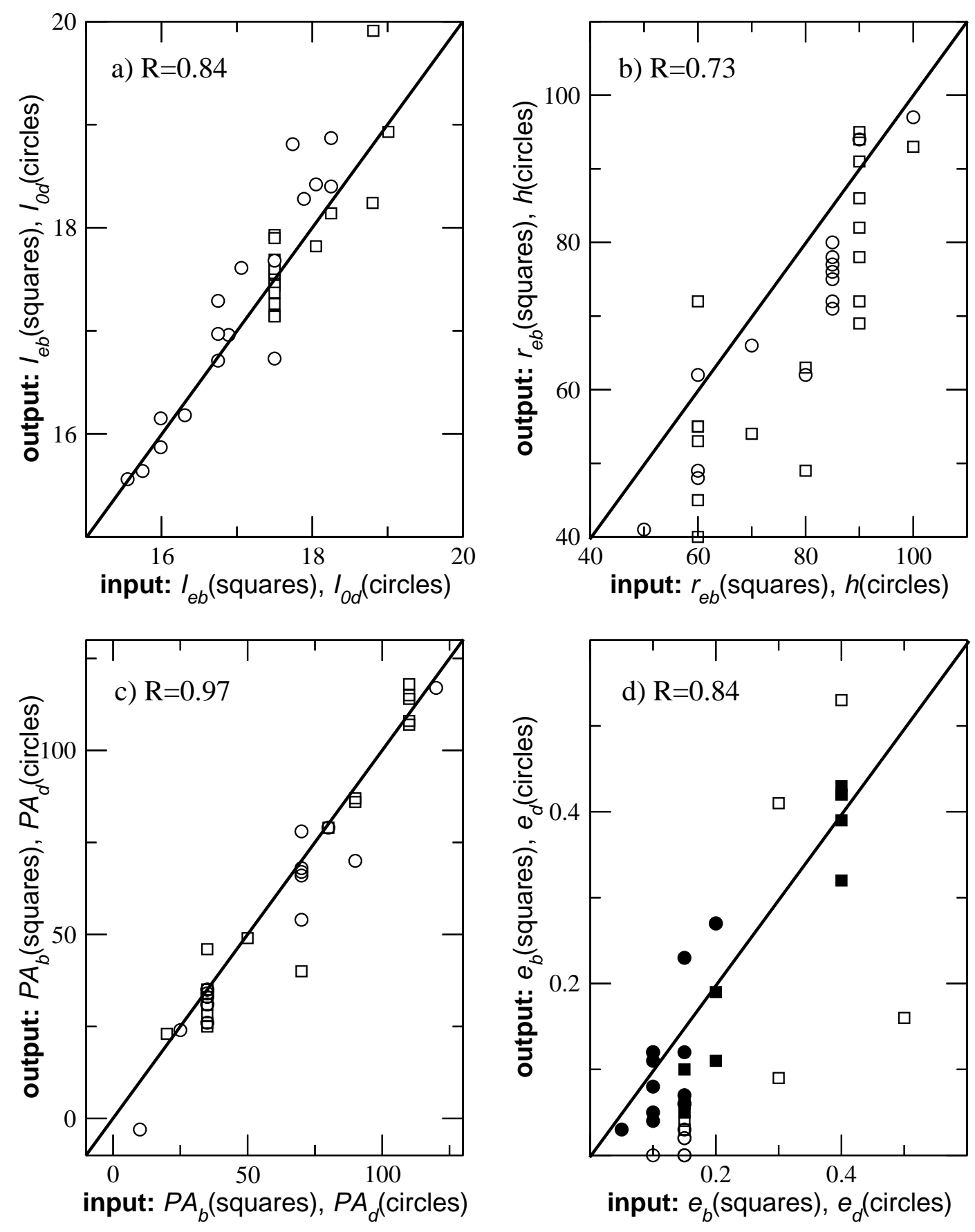

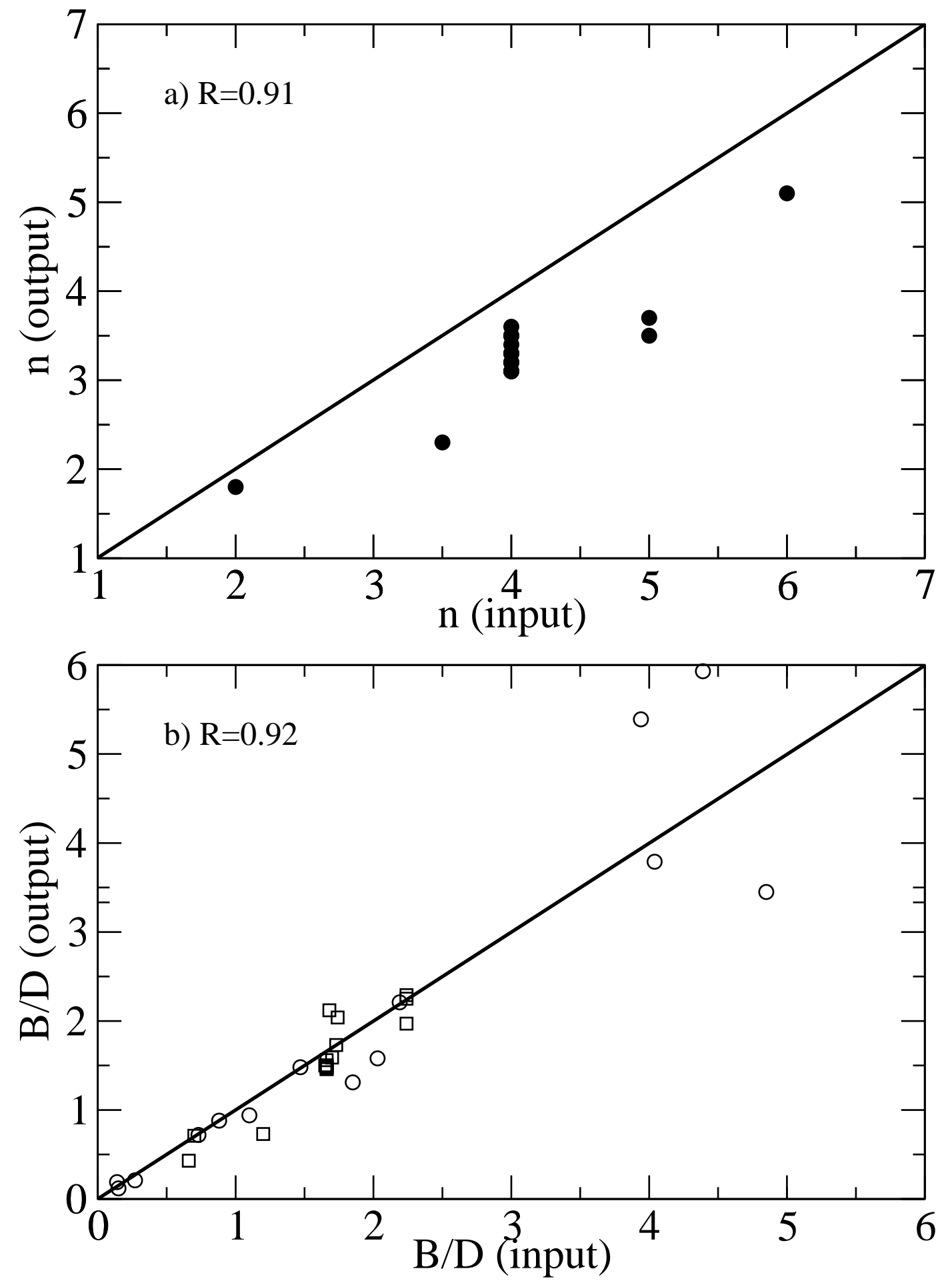


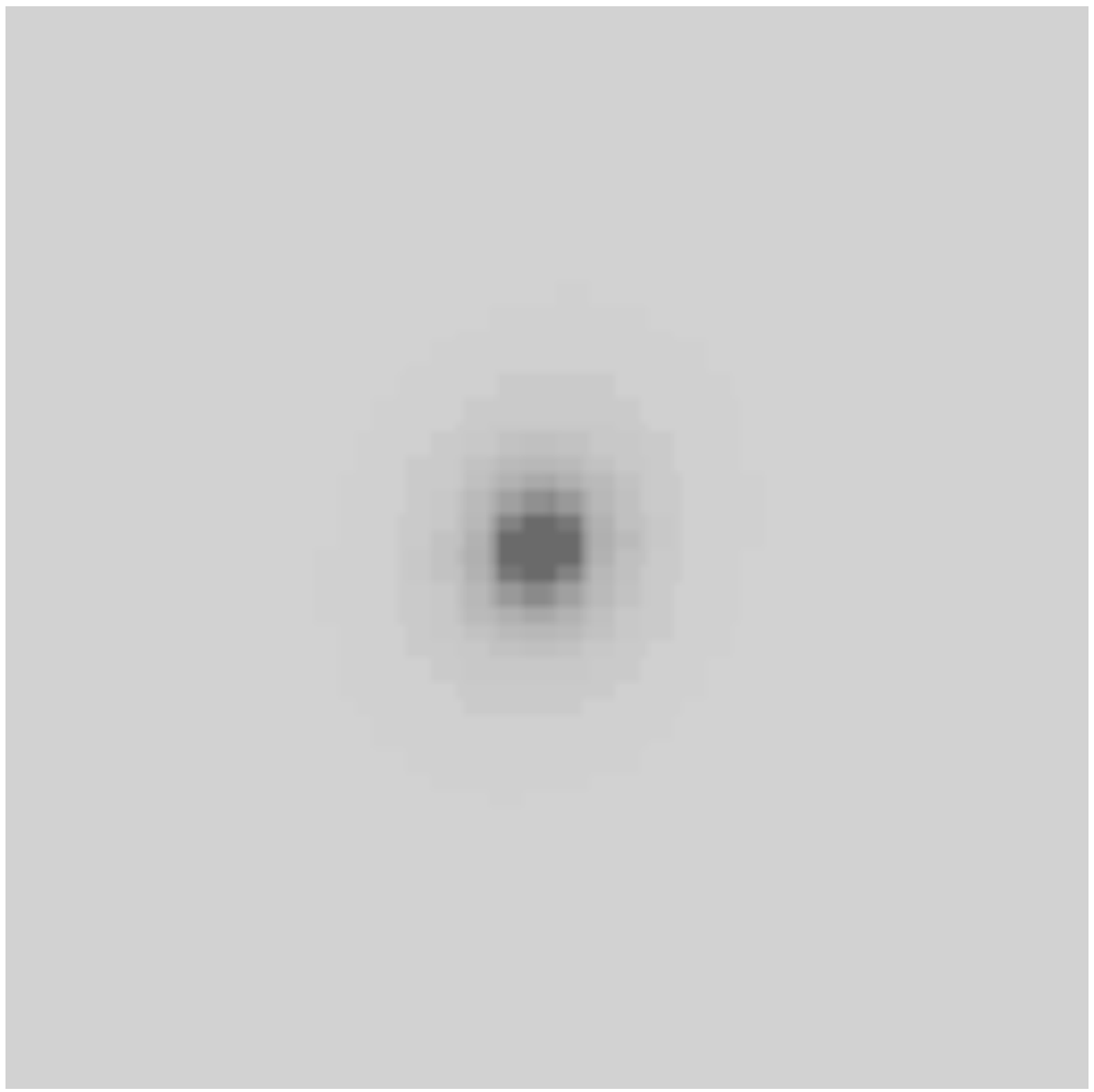




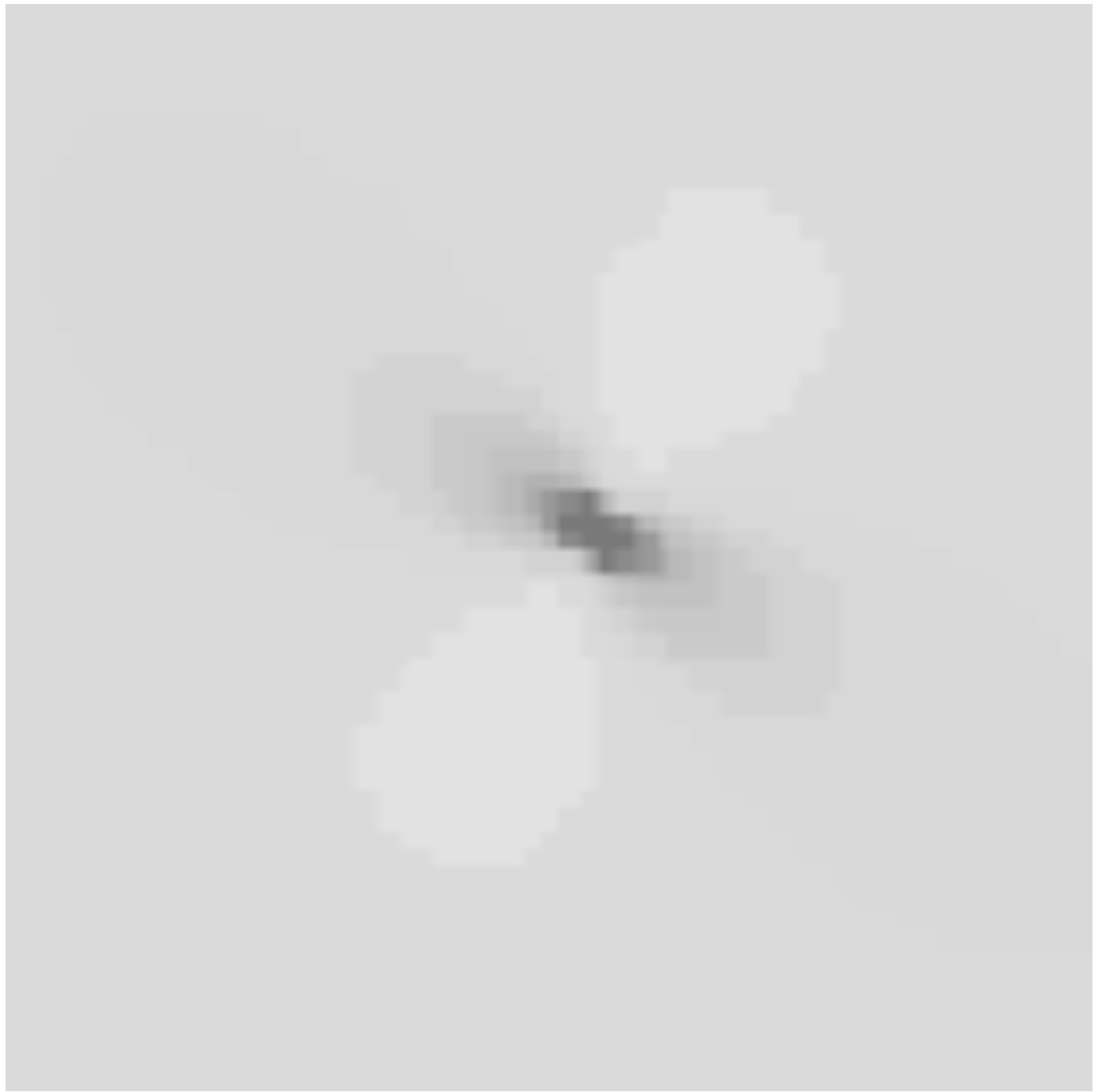



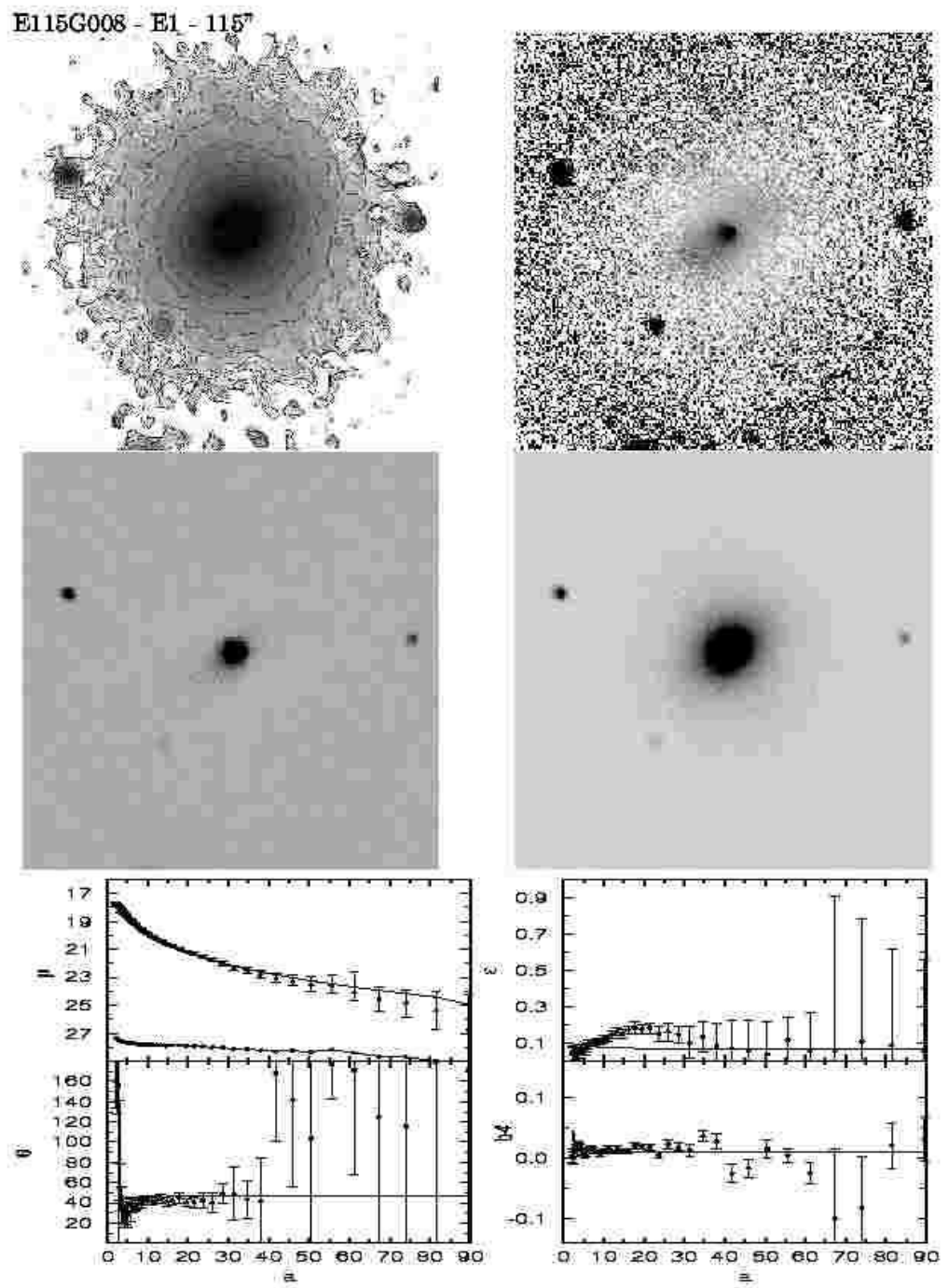

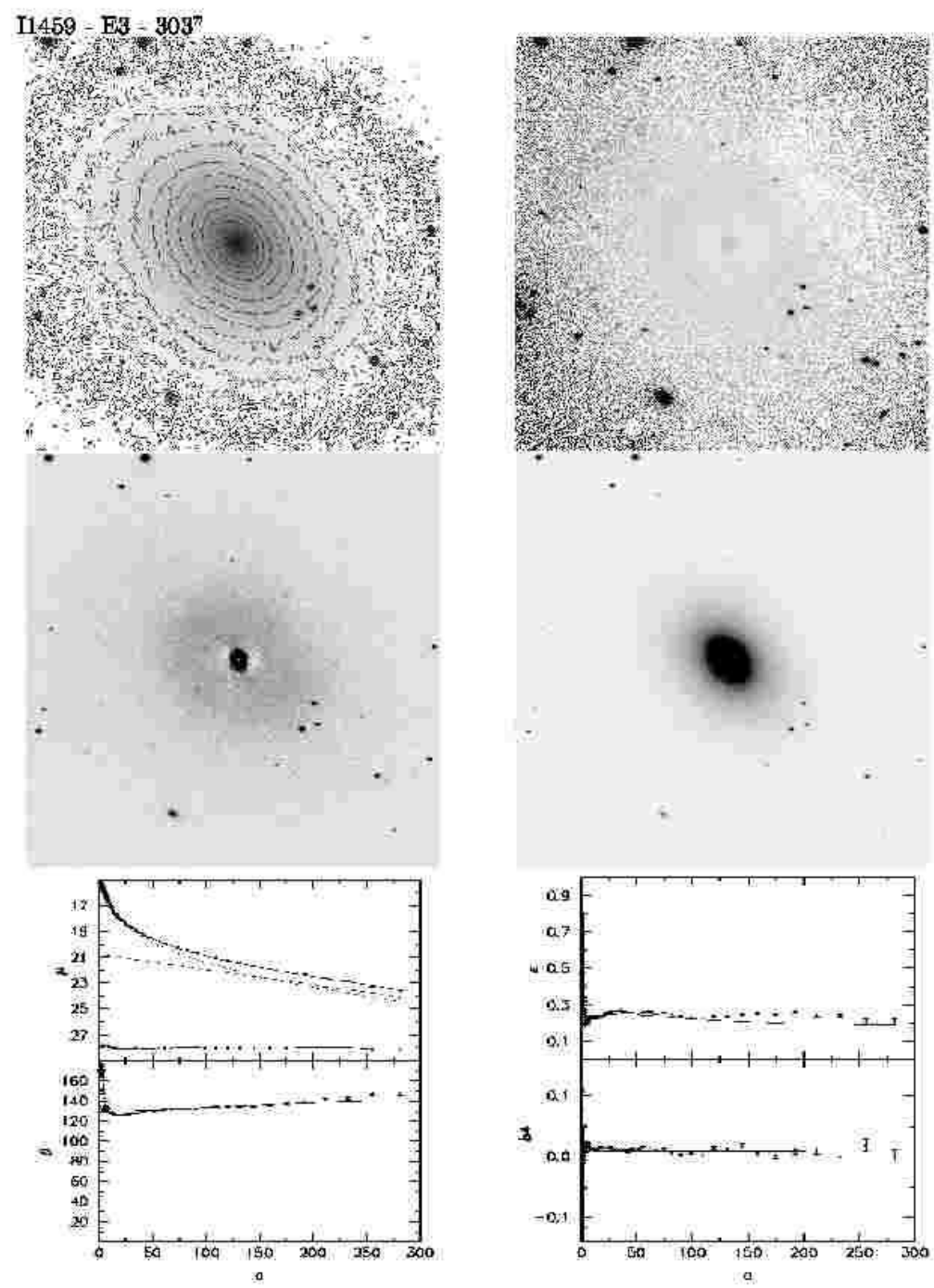

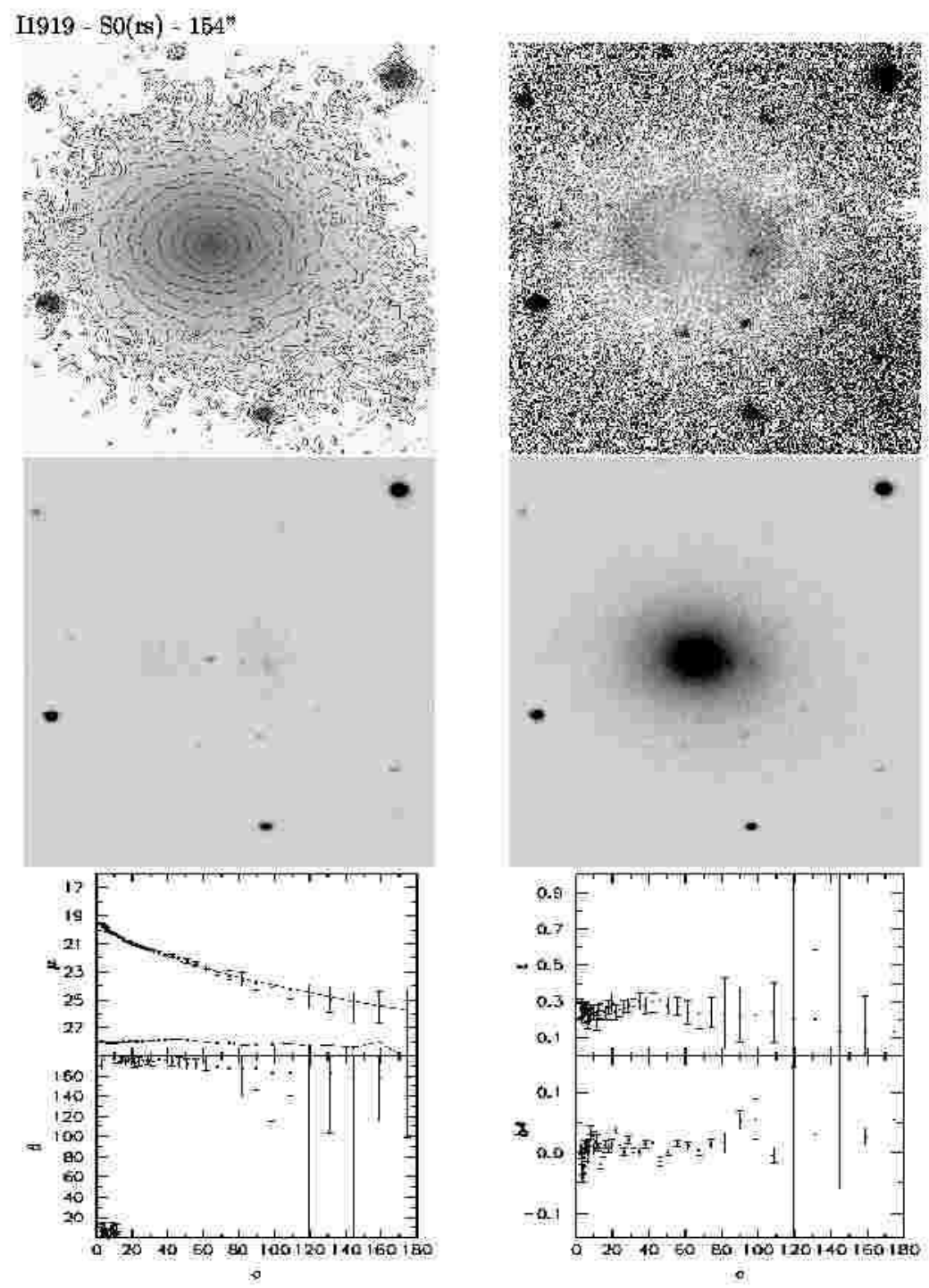

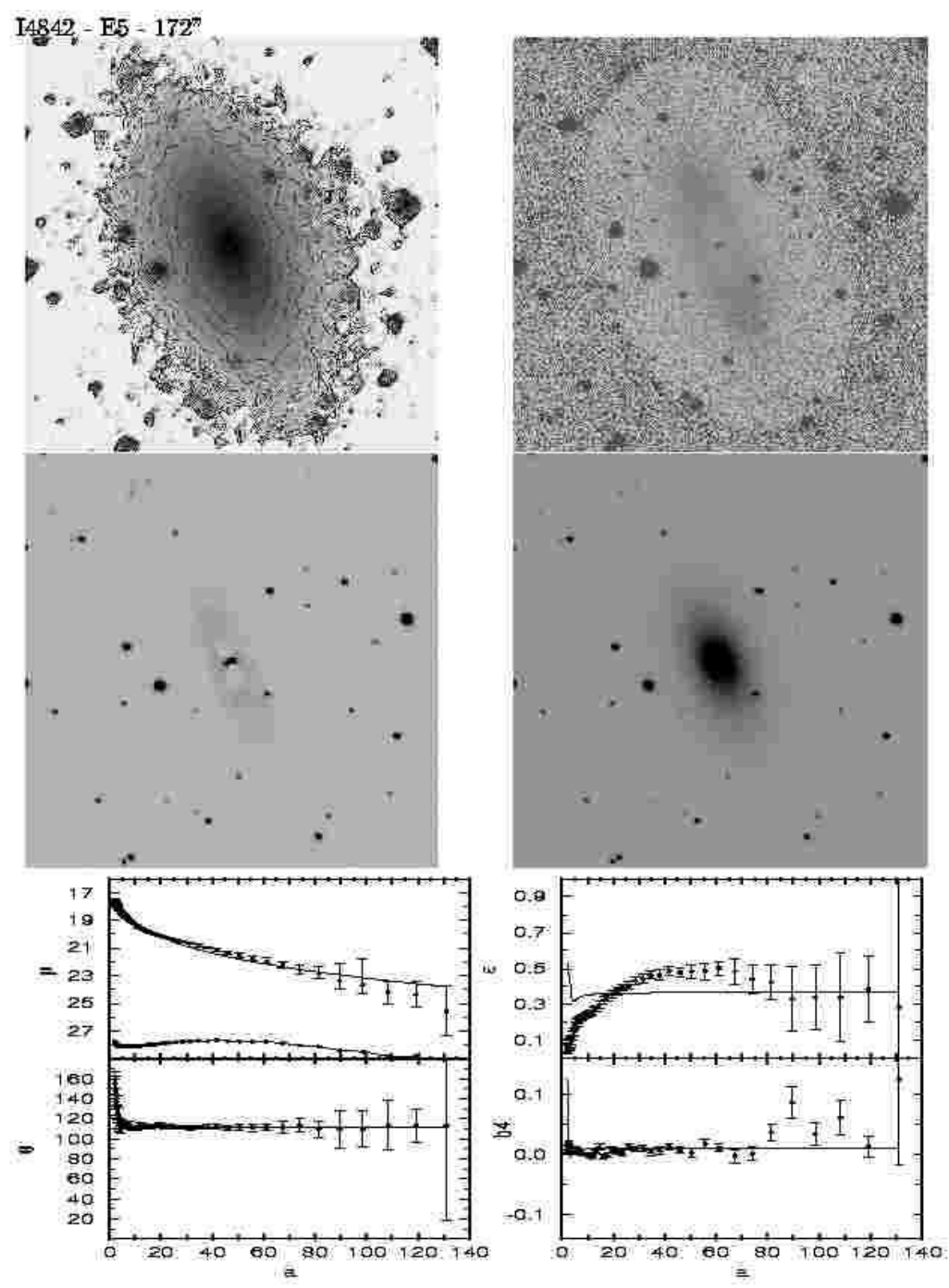

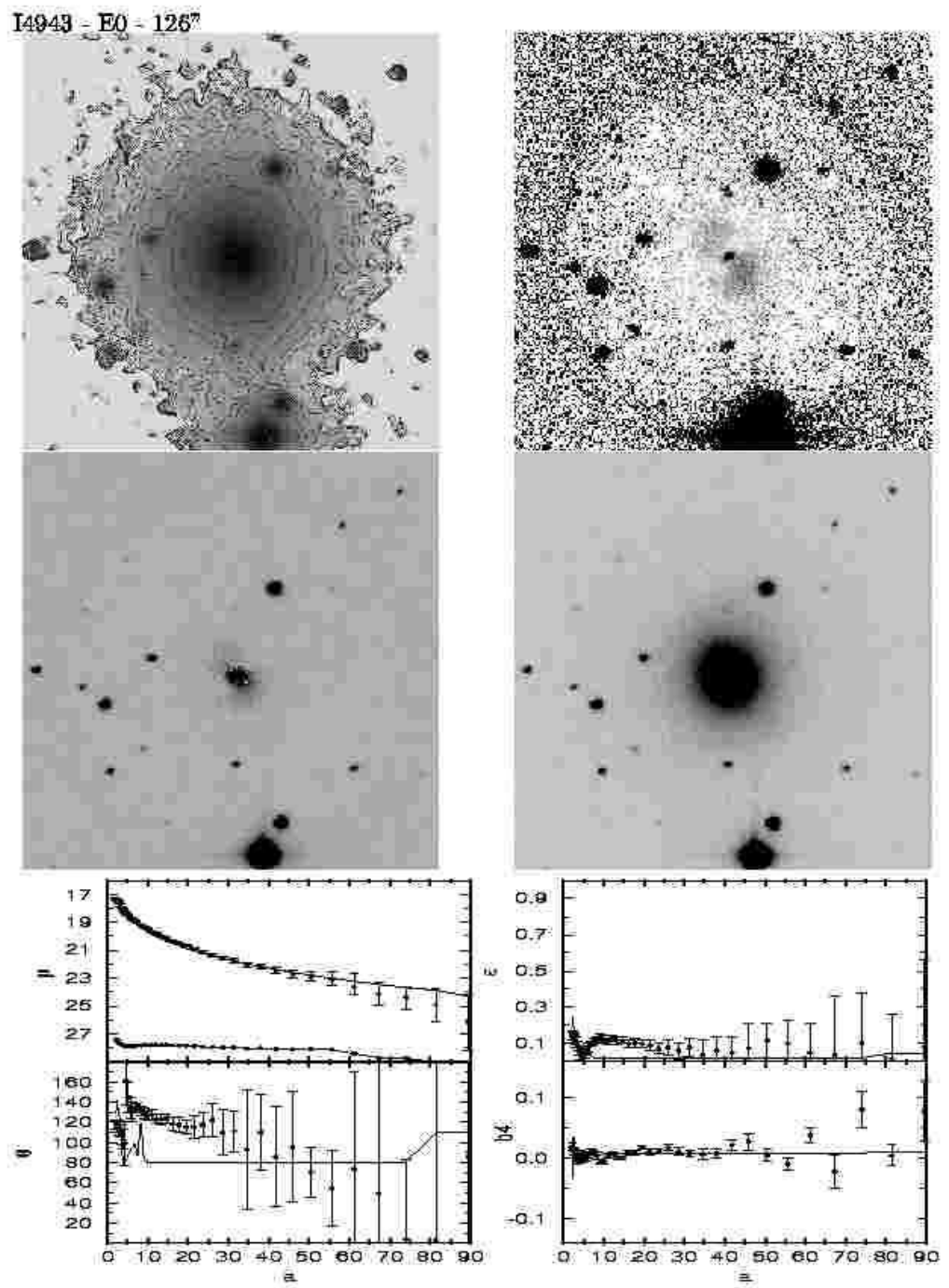
Table 1. Results from the structural analysis for 51 galaxies.

\begin{tabular}{|c|c|c|c|c|c|c|c|c|c|}
\hline Galaxy & $\begin{array}{c}\mathrm{T} \\
(\mathrm{RC} 3)\end{array}$ & $\begin{array}{c}\text { B/D } \\
\text { (Luminosity) }\end{array}$ & $\begin{array}{c}\text { B/D } \\
\text { (Size) }\end{array}$ & $\begin{array}{c}I_{0 d} \\
\left(\mathrm{R} \mathrm{mag} \operatorname{arcsec}^{-2}\right)\end{array}$ & $\begin{array}{c}h^{\mathrm{a}} \\
(\operatorname{arcsec})\end{array}$ & $\begin{array}{c}I_{e b} \\
\left(\mathrm{R} \operatorname{mag} \operatorname{arcsec}^{-2}\right)\end{array}$ & $\begin{array}{c}r_{e b}{ }^{\mathrm{a}} \\
(\operatorname{arcsec})\end{array}$ & $n^{\mathrm{a}}$ & Comments \\
\hline ESO115G008 & E1 & $\ldots$ & $\ldots$ & $\ldots$ & $\ldots$ & 21.02 & $18.30 \pm 1.10$ & $4.67 \pm 0.69$ & inner disk \\
\hline IC1919 & $\mathrm{S} 0(\mathrm{rs})$ & $\ldots$ & $\ldots$ & $\ldots$ & $\ldots$ & 21.31 & $31.12 \pm 1.56$ & $1.87 \pm 0.24$ & trueE \\
\hline IC4842 & E5 & $\ldots$ & $\ldots$ & $\ldots$ & $\ldots$ & 21.65 & $49.04 \pm 2.71$ & $4.79 \pm 0.57$ & $\ldots$ \\
\hline IC4943 & E0 & $\ldots$ & $\ldots$ & $\ldots$ & $\ldots$ & 20.96 & $21.96 \pm 0.49$ & $5.28 \pm 0.32$ & $\ldots$ \\
\hline IC5250A & So & 0.43 & 1.10 & 20.15 & $67.26 \pm 22.85$ & 20.59 & $15.09 \pm 2.60$ & $4.58 \pm 2.09$ & $\ldots$ \\
\hline NGC0467 & So & 3.62 & 1.48 & 20.95 & $34.17 \pm 3.64$ & 20.77 & $30.99 \pm 0.50$ & $3.54 \pm 0.13$ & $\ldots$ \\
\hline NGC0541 & So & $\ldots$ & $\ldots$ & $\ldots$ & $\ldots$ & 21.33 & $35.84 \pm 1.54$ & $3.93 \pm 0.38$ & trueE \\
\hline NGC0720 & E5 & $\ldots$ & $\ldots$ & $\ldots$ & $\ldots$ & 20.01 & $58.20 \pm 2.17$ & $3.86 \pm 0.34$ & $\ldots$ \\
\hline NGC0821 & E6 & $\ldots$ & $\ldots$ & $\ldots$ & $\ldots$ & 20.78 & $56.56 \pm 3.77$ & $4.42 \pm 0.67$ & inner disk \\
\hline NGC0822 & E3 & $\ldots$ & $\ldots$ & $\ldots$ & $\ldots$ & 20.66 & $18.69 \pm 0.59$ & $4.34 \pm 0.36$ & $\ldots$ \\
\hline NGC1052 & E4 & 13.83 & 1.70 & 18.81 & $19.27 \pm 2.37$ & 20.02 & $47.76 \pm 0.85$ & $4.75 \pm 0.21$ & inner disk \\
\hline NGC1079 & $\mathrm{SAB} 0 / \mathrm{a}(\mathrm{rs})$ & 100.24 & 1.89 & 21.53 & $13.40 \pm 22.54$ & 20.78 & $50.86 \pm 2.26$ & $3.82 \pm 0.41$ & $\ldots$ \\
\hline NGC1172 & E3 & 2.81 & 1.50 & 21.60 & $92.91 \pm 63.22$ & 21.14 & $31.79 \pm 2.27$ & $5.02 \pm 0.76$ & trueSO \\
\hline NGC1199 & E3 & $\ldots$ & $\ldots$ & $\ldots$ & $\ldots$ & 20.41 & $35.54 \pm 0.92$ & $3.89 \pm 0.24$ & $\ldots$ \\
\hline NGC1291 & $\mathrm{SB} 0 / \mathrm{a}(\mathrm{s})$ & 2.63 & 1.48 & 17.75 & $35.35 \pm 3.14$ & 19.56 & $55.88 \pm 2.58$ & $4.63 \pm 0.51$ & inner bar/ring \\
\hline NGC1316 & SAB0(s) & 4.51 & 2.13 & 19.45 & $71.02 \pm 5.63$ & 19.69 & $89.23 \pm 2.29$ & $4.67 \pm 0.28$ & dust lane \\
\hline NGC1326 & SB0(r) & 4.11 & 1.73 & 19.91 & $32.63 \pm 7.59$ & 20.02 & $38.54 \pm 2.23$ & $4.89 \pm 0.71$ & inner ring \\
\hline NGC1549 & E0 & 9.52 & 1.45 & 19.47 & $27.90 \pm 6.10$ & 19.70 & $52.25 \pm 2.22$ & $4.42 \pm 0.44$ & trueS0+lens \\
\hline NGC1553 & S0 & $\ldots$ & $\ldots$ & $\ldots$ & $\ldots$ & 20.28 & $81.01 \pm 6.86$ & $5.24 \pm 0.90$ & trueE+lens \\
\hline NGC1637 & $\mathrm{SABc}(\mathrm{rs})$ & 0.24 & 0.63 & 18.91 & $36.56 \pm 4.49$ & 20.31 & $18.96 \pm 6.11$ & $3.18 \pm 1.97$ & $\ldots$ \\
\hline NGC1700 & $\mathrm{E} 4$ & 22.46 & 1.68 & 18.75 & $9.61 \pm 1.04$ & 20.05 & $36.50 \pm 0.34$ & $3.89 \pm 0.08$ & inner disk \\
\hline NGC1947 & S0 & 9.44 & 1.44 & 22.62 & $33.03 \pm 12.94$ & 22.17 & $49.58 \pm 2.08$ & $2.81 \pm 0.23$ & dust lane \\
\hline NGC2205 & SAB0(rs) & $\ldots$ & $\ldots$ & $\ldots$ & $\ldots$ & 22.27 & $23.62 \pm 0.94$ & $4.43 \pm 0.44$ & trueE \\
\hline NGC2217 & $\mathrm{SB} 0(\mathrm{rs})$ & $\ldots$ & $\ldots$ & $\ldots$ & $\ldots$ & 22.24 & $59.01 \pm 7.15$ & $4.40 \pm 1.48$ & no disk \\
\hline NGC2271 & SAB0 & $\ldots$ & $\ldots$ & $\ldots$ & $\ldots$ & 22.00 & $28.94 \pm 1.02$ & $2.81 \pm 0.25$ & trueE+inner disk \\
\hline NGC2293 & $\mathrm{SAB0}(\mathrm{s})$ & 1.41 & 1.91 & 21.87 & $72.14 \pm 17.57$ & 21.47 & $28.67 \pm 2.46$ & $4.06 \pm 0.94$ & $\ldots$ \\
\hline NGC2305 & E2 & 2.80 & 1.84 & 22.49 & $44.10 \pm 14.73$ & 21.03 & $19.75 \pm 1.09$ & $2.99 \pm 0.45$ & trueso \\
\hline NGC6578 & E2 & $\ldots$ & $\ldots$ & $\ldots$ & $\ldots$ & 20.80 & $43.11 \pm 2.58$ & $4.84 \pm 0.72$ & $\ldots$ \\
\hline NGC6673 & SAB0(s) & 7.92 & 2.06 & 20.69 & $33.72 \pm 34.14$ & 20.19 & $34.60 \pm 3.16$ & $5.07 \pm 1.11$ & $\ldots$ \\
\hline NGC6849 & SB0 & $\ldots$ & $\ldots$ & $\ldots$ & $\ldots$ & 21.73 & $51.57 \pm 2.62$ & $4.12 \pm 0.42$ & trueE \\
\hline NGC6958 & $\mathrm{E} 2$ & $\ldots$ & $\ldots$ & $\ldots$ & $\ldots$ & 20.18 & $36.98 \pm 2.17$ & $4.49 \pm 0.68$ & $\ldots$ \\
\hline NGC7070A & Irr & 1.26 & 1.64 & 19.75 & $20.43 \pm 2.73$ & 20.92 & $22.65 \pm 2.04$ & $2.74 \pm 0.48$ & dust lane \\
\hline NGC7145 & E0 & 4.65 & 1.44 & 20.88 & $41.37 \pm 7.14$ & 21.06 & $44.35 \pm 1.25$ & $4.88 \pm 0.29$ & trueS0+lens \\
\hline
\end{tabular}


Table 1-Continued

\begin{tabular}{|c|c|c|c|c|c|c|c|c|c|}
\hline Galaxy & $\begin{array}{c}\mathrm{T} \\
(\mathrm{RC} 3)\end{array}$ & $\begin{array}{c}\text { B/D } \\
\text { (Luminosity) }\end{array}$ & $\begin{array}{l}\text { B/D } \\
(\text { Size })\end{array}$ & $\begin{array}{c}I_{0 d} \\
\left(\mathrm{R} \operatorname{mag} \operatorname{arcsec}^{-2}\right)\end{array}$ & $\begin{array}{c}h^{\mathrm{a}} \\
(\operatorname{arcsec})\end{array}$ & $\begin{array}{c}I_{e b} \\
\left(\mathrm{R} \mathrm{mag} \operatorname{arcsec}^{-2}\right)\end{array}$ & $\begin{array}{c}r_{e b^{\mathrm{a}}} \\
(\operatorname{arcsec})\end{array}$ & $n^{\mathrm{a}}$ & Comments \\
\hline NGC7171 & $\mathrm{SBb}(\mathrm{rs})$ & 0.44 & 1.12 & 20.18 & $41.28 \pm 8.00$ & 21.25 & $40.57 \pm 10.18$ & $2.17 \pm 1.38$ & \\
\hline NGC7177 & $\mathrm{SABb}(\mathrm{r})$ & 7.55 & 1.63 & 18.58 & $12.97 \pm 4.07$ & 20.19 & $34.89 \pm 2.86$ & $3.52 \pm 0.68$ & inner ring \\
\hline NGC7192 & E0 & 118.52 & 1.44 & 21.16 & $7.98 \pm 10.44$ & 20.61 & $39.05 \pm 1.22$ & $4.06 \pm 0.30$ & inner disk \\
\hline NGC7196 & E2 & $\ldots$ & $\ldots$ & $\ldots$ & $\ldots$ & 20.05 & $30.65 \pm 1.45$ & $4.05 \pm 0.48$ & inner disk \\
\hline NGC7252 & S0 & $\ldots$ & $\ldots$ & $\ldots$ & $\ldots$ & 19.92 & $19.72 \pm 0.99$ & $4.03 \pm 0.55$ & trueE? \\
\hline NGC7280 & $\mathrm{SAB} 0(\mathrm{r})$ & 2.96 & 2.07 & 20.34 & $27.47 \pm 4.94$ & 20.76 & $28.78 \pm 1.41$ & $5.32 \pm 0.67$ & lens \\
\hline NGC7289 & $\mathrm{S} 0(\mathrm{r})$ & $\ldots$ & $\ldots$ & $\ldots$ & $\ldots$ & 21.86 & $33.61 \pm 2.15$ & $4.56 \pm 0.63$ & trueE \\
\hline NGC7371 & $\mathrm{S} 0 / \mathrm{a}(\mathrm{r})$ & 1.10 & 1.46 & 20.00 & $35.49 \pm 2.48$ & 21.98 & $35.72 \pm 1.72$ & $3.27 \pm 0.37$ & bar \\
\hline NGC7377 & $\mathrm{S} 0(\mathrm{~s})$ & 2.62 & 1.63 & 21.09 & $75.53 \pm 19.66$ & 20.87 & $52.63 \pm 2.00$ & $4.01 \pm 0.33$ & $\ldots$ \\
\hline NGC7507 & E0 & $\ldots$ & $\ldots$ & $\ldots$ & $\ldots$ & 20.08 & $46.97 \pm 1.46$ & $4.35 \pm 0.35$ & $\ldots$ \\
\hline NGC7619 & $\mathrm{E} 2$ & $\ldots$ & $\ldots$ & $\ldots$ & $\ldots$ & 20.51 & $41.79 \pm 0.78$ & $4.34 \pm 0.19$ & $\ldots$ \\
\hline NGC7778 & E0 & 1.34 & 1.36 & 21.10 & $55.86 \pm 0.29$ & 19.75 & $11.40 \pm 0.29$ & $3.40 \pm 0.10$ & trueS0 \\
\hline NGC7824 & Sab & $\ldots$ & $\ldots$ & $\ldots$ & $\ldots$ & 20.70 & $21.32 \pm 1.02$ & $4.47 \pm 0.50$ & no disk? \\
\hline PGC64863 & $\mathrm{E} 4$ & $\ldots$ & $\ldots$ & $\ldots$ & $\ldots$ & 21.33 & $19.37 \pm 1.43$ & $3.29 \pm 0.50$ & inner disk \\
\hline PGC67633 & S0 & $\ldots$ & $\ldots$ & $\ldots$ & $\ldots$ & 21.80 & $21.88 \pm 2.05$ & $4.32 \pm 0.88$ & trueE \\
\hline
\end{tabular}

${ }^{\mathrm{a}}$ Errors shown correspond to $1 \sigma$. 